\title{
Nanoclustering phase competition induces the resistivity hump in colossal magnetoresistive manganites
}

\author{
Kalpataru Pradhan ${ }^{1,2 *}$ and Seiji Yunoki ${ }^{2,3,4 \dagger}$ \\ ${ }^{1}$ CMP Division, Saha Institute of Nuclear Physics, HBNI, Kolkata 700064, India \\ ${ }^{2}$ Computational Quantum Matter Research Team, RIKEN, \\ Center for Emergent Matter Science (CEMS), Saitama 351-0198, Japan \\ ${ }^{3}$ Computational Condensed Matter Physics Laboratory, RIKEN, Wako, Saitama 351-0198, Japan \\ ${ }^{4}$ Computational Materials Science Research Team, \\ RIKEN Advanced Institute for Computational Science (AICS), Hyogo 650-0047, Japan
}

(Dated: November 8, 2018)

\begin{abstract}
Using a two-band double-exchange model with Jahn-Teller lattice distortions and super-exchange interactions, supplemented by quenched disorder, at electron density $n=0.65$, we explicitly demonstrate the coexistence of the $n=1 / 2$-type $(\pi, \pi)$ charge-ordered and the ferromagnetic nanoclusters above the ferromagnetic transition temperature $T_{\mathrm{c}}$ in colossal magnetoresistive $(\mathrm{CMR})$ manganites. The resistivity increases due to the enhancement of the volume fraction of the charge-ordered and the ferromagnetic nanoclusters with decreasing the temperature down to $T_{\mathrm{c}}$. The ferromagnetic nanoclusters start to grow and merge, and the volume fraction of the charge-ordered nanoclusters decreases below $T_{\mathrm{c}}$, leading to the sharp drop in the resistivity. By applying a small external mag-

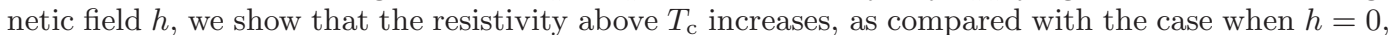
a fact which further confirms the coexistence of the charge-ordered and the ferromagnetic nanoclusters. In addition, we show that the volume fraction of the charge-ordered nanoclusters decreases with increasing the bandwidth and consequently the resistivity hump diminishes for large bandwidth manganites, in good qualitative agreement with experiments. The obtained insights from our calculations provide a complete pathway to understand the phase competition in CMR manganites.
\end{abstract}

\section{INTRODUCTION}

The strong coupling between charge, spin, orbital, and lattice degrees of freedom, supplemented by weak disorder, leads to unusual colossal response phenomena in manganites ${ }^{1-4}$. There has been intense focus over last two decades to validate the phase coexistence/competition scenario ${ }^{5-18}$, which is believed to be a necessary ingredient to explain the resistivity hump in colossal magnetoresistive (CMR) manganites. Furthermore, understanding the phase competition in bulk manganites helps in designing low-dimensional manganite nanostructures with emergent physical phenomena ${ }^{19-21}$.

The manganites, materials of the form $\mathrm{R}_{1-x} \mathrm{~A}_{x} \mathrm{MnO}_{3}$ where $\mathrm{R}$ is rare-earth (La, $\mathrm{Nd}$, Pr, etc.) and $\mathrm{A}$ is alkalineearth $(\mathrm{Ca}, \mathrm{Sr}$, and $\mathrm{Ba})$ elements, are best known for their CMR property in the doping range $x=1-n=0.3-0.4$ ( $n$ : electron density). The bandwidth of the manganites increases with the mean radius $r_{\mathrm{A}}$ of $\mathrm{R}$ and $\mathrm{A}$ ions ${ }^{22,23}$, and the size mismatch between the two radii controls the strength of the cation disorder ${ }^{24,25}$. Figure 1(a), reproduced from Ref. 23, schematically summarizes a phase diagram of the manganites. Low temperature magnetic states for $n=0.5$ and 0.65 are also listed in Table I along with ionic radii of different $\mathrm{R}$ and $\mathrm{A}$ ions.

The phase competition arising due to the proximity of variety of phases, in presence of cationic disorder, often leads to novel phenomena such as inhomogeneities, phase coexistence, and percolative transport ${ }^{3,4}$. Recent experiments $^{15-18}$ show that nanoscale ferromagnetic (FM) regions and $n=1 / 2$-type $(\pi, \pi)$ charge-ordered $(\mathrm{CO})^{5}$ regions, which characterizes the CE-type phase, coexist above the FM transition temperature $T_{\mathrm{c}}$. The precise microscopic origin of the phase competition above $T_{\mathrm{c}}$ is still elusive theoretically ${ }^{26,27}$. The absence of FM correlations above $T_{\mathrm{c}}$ fails to establish the phase competition scenario in CMR manganites in recent theoretical stud$\operatorname{ies}^{28,29}$.

In this paper, we explicitly demonstrate the phase competition between the $n=1 / 2$-type $(\pi, \pi) \mathrm{CO}$ nanoclusters and the FM nanoclusters at $n=0.65$, relevant to the CMR manganites, above the FM $T_{\mathrm{c}}$. Our study reveals that this phase competition induces the resistivity hump, and is expected only in those manganites for which the ground state is the CE-type phase at $n \sim 0.5$. With increasing the bandwidth, the volume fraction of the $\mathrm{CO}$ nanoclusters decreases and consequently the resistivity hump around $T_{\mathrm{c}}$ vanishes.

\section{MODEL HAMILTONIAN AND METHOD}

We consider the following two-band model Hamiltonian $^{3}$, a minimal model that is required to capture the essential phases in manganites ${ }^{30-33}$, in two spatial dimensions:

$$
\begin{aligned}
H= & -\sum_{\langle i j\rangle, s} \sum_{\alpha, \beta} t_{\alpha \beta}^{i j} c_{i \alpha s}^{\dagger} c_{j \beta s}-J_{H} \sum_{i} \mathbf{S}_{i} \cdot \boldsymbol{\sigma}_{i}+J \sum_{\langle i j\rangle} \mathbf{S}_{i} \cdot \mathbf{S}_{j} \\
& -\lambda \sum_{i} \mathbf{Q}_{i} \cdot \boldsymbol{\tau}_{i}+\frac{K}{2} \sum_{i} \mathbf{Q}_{i}^{2}+\sum_{i}\left(\epsilon_{i}-\mu\right) n_{i},
\end{aligned}
$$

where $c_{i \alpha s}^{\dagger}$ is the electron creation operator at site $i$ with orbital $\alpha=(a, b)$ and spin $s(=\uparrow, \downarrow)$. The kinetic energy term includes inter- and intra-orbital nearest-neighbor 

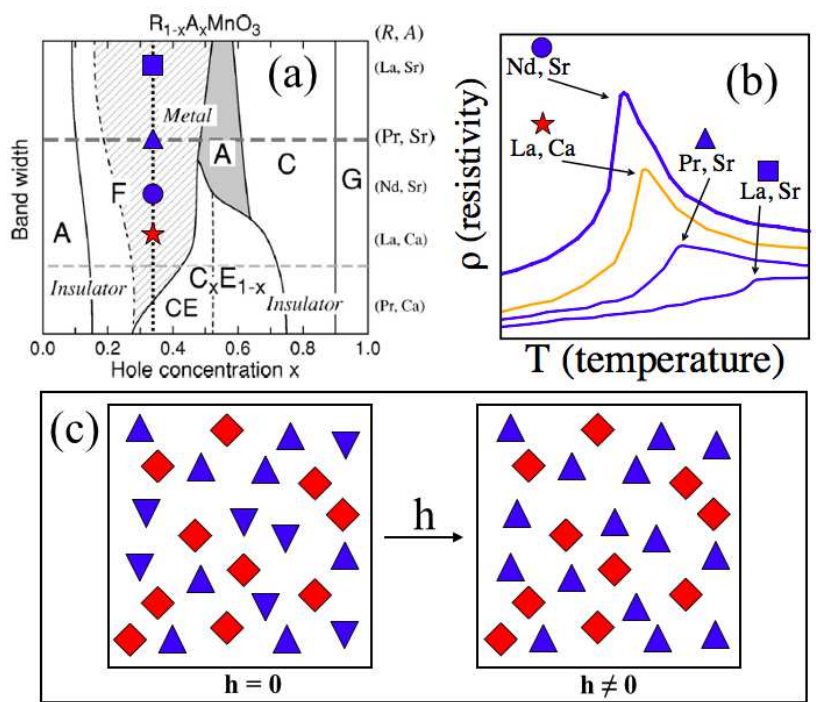

FIG. 1. (Color online) (a) Schematic low-temperature phase diagram of manganites $\mathrm{R}_{1-x} \mathrm{~A}_{x} \mathrm{MnO}_{3}$ for different $\mathrm{R}$ (rare-earth) and A (alkaline-earth) elements (adopted from Ref. 23). F, A and CE-type phases near $x=1-n=0.35$ are of interests to the present study and they denote ferromagnetic (FM), A-type antiferromagnetic, and CE-type antiferromagnetic phases, respectively. (b) Schematic to illustrate the temperature dependence of the resistivity for different bandwidth manganites at $x=1-n \sim 1 / 3$. Same symbols in (a) and (b) are used to indicate the combination of $\mathrm{R}$ and $\mathrm{A}$. (c) Schematic to show the phase coexistence between chargeordered (red diamonds) and ferromagnetic (blue triangles) nanoclusters without and with a small external magnetic field $h$ above the FM $T_{\mathrm{c}}$. The direction of a triangle implies the overall spin orientation in each FM nanoclusters (see the text for details).

hopping $t_{\alpha \beta}^{i j}$, i.e. $t_{a a}^{x}=t_{a a}^{y} \equiv t, t_{b b}^{x}=t_{b b}^{y} \equiv t / 3$, $t_{a b}^{x}=t_{b a}^{x} \equiv-t / \sqrt{3}$, and $t_{a b}^{y}=t_{b a}^{y} \equiv t / \sqrt{3}$ along $x$ and $y$ directions, where $a$ and $b$ refer to two Mn $e_{g}$ orbitals $d_{x^{2}-y^{2}}$ and $d_{3 z^{2}-r^{2}}$, respectively. Hund's coupling $J_{H}$ is between $t_{2 g}$ spin $\mathbf{S}_{i}$ and $e_{g}$ electron spin $\boldsymbol{\sigma}_{i}$ at site $i$. Here, we adopt the double-exchange limit, i.e. $J_{H} \rightarrow \infty$, as $t(\sim 0.2-0.5 \mathrm{eV})$ is estimated to be much smaller than $J_{H}(\sim 2 \mathrm{eV})^{3} . J$ is the antiferromagnetic super-exchange between nearest neighboring $t_{2 g}$ spins. $\lambda$ represents the electron-phonon coupling between Jahn-Teller phonons $\mathbf{Q}_{i}$ and $e_{g}$ electrons in the adiabatic limit. We treat all $\mathbf{S}_{i}$ and $\mathbf{Q}_{i}$ as classical variables ${ }^{34-36}$, and the stiffness $K$ of Jahn-Teller modes and $\left|\mathbf{S}_{i}\right|$ are set to be 1 . We also choose a typical value of $J / t=0.1^{30}$. Effects of disorder is taken into account by the $\sum_{i} \epsilon_{i} n_{i}$ term where $\epsilon_{i}$ is the quenched binary disorder potential with values $\pm \Delta$. $\mu$ is the chemical potential. To analyze the effect of external magnetic field $\mathbf{h}=h \hat{\mathbf{z}}$, wherever necessary, we add a Zeeman coupling term $-\sum_{i} \mathbf{h} \cdot \mathbf{S}_{i}$ to the Hamiltonian. We measure $\lambda, \Delta, h$ and temperature $T$ in units of $t$.

An exact diagonalization scheme is applied to the mobile $e_{g}$ electrons in the background of classical spins $\mathbf{S}_{i}$

\begin{tabular}{|c|c||c|c||c|c|}
\hline \multicolumn{3}{|c||}{ experimentally observed magnetic phases } & \multicolumn{2}{c|}{ parameters } \\
\hline manganites & radii $(\mathrm{R}, \mathrm{A})$ in $\AA$ & $\mathrm{n}=0.65$ & $\mathrm{n}=0.5$ & $\lambda$ & $\Delta$ \\
\hline PCMO & $1.29,1.34$ & CE-type & CE-type & 1.75 & 0.1 \\
\hline LCMO & $1.36,1.34$ & FM-M & CE-type & 1.70 & 0.05 \\
\hline NSMO & $1.27,1.44$ & FM-M & CE-type & 1.65 & 0.2 \\
\hline PSMO & $1.29,1.44$ & FM-M & A-type & 1.55 & 0.2 \\
\hline LSMO & $1.36,1.44$ & FM-M & FM-M & 1.50 & 0.1 \\
\hline
\end{tabular}

TABLE I. Magnetic states observed experimentally at low temperatures [from Fig. 1(a)] for $\mathrm{Pr}_{1-x} \mathrm{Ca}_{x} \mathrm{MnO}_{3}$ (PCMO), $\mathrm{La}_{1-x} \mathrm{Ca}_{x} \mathrm{MnO}_{3}$ (LCMO), $\mathrm{Nd}_{1-x} \mathrm{Sr}_{x} \mathrm{MnO}_{3} \quad$ (NSMO), $\mathrm{Pr}_{1-x} \mathrm{Sr}_{x} \mathrm{MnO}_{3}$ (PSMO), and $\mathrm{La}_{1-x} \mathrm{Sr}_{x} \mathrm{MnO}_{3}$ (LSMO) at $n=1-x=0.65$ and 0.5. FM-M stands for FM metal. $\lambda$ and $\Delta$ values used in our calculations to qualitatively reproduce the experimental results and ionic radii of $\mathrm{R}$ and $\mathrm{A}$ elements ${ }^{4}$ are also given.

and lattice distortions $\mathbf{Q}_{i}$, and a Monte Carlo method is employed for classical variables. This spin-fermion Monte Carlo method adequately takes spatial fluctuations into account that is necessary to study the inhomogeneities. In order to handle large system sizes (up to $N=24 \times 24$ sites), we employ a Monte Carlo technique based on traveling cluster approximation ${ }^{31,33,37}$. The randomized classical spins $\mathbf{S}_{i}$ and lattice distortions $\mathbf{Q}_{i}$ are annealed starting from a high temperature in an arbitrary quenched disorder configuration. All physical quantities such as magnetization and resistivity are averaged over ten such different disorder configurations in addition to the thermal averages taken during the Monte Carlo simulations.

\section{PHASE DIAGRAM $(\lambda=1.7$ AND $J=0.1)$}

Figures 2(a) and 2(b) show that the ground state is FM metallic in $e_{g}$ electron density $n=0.63-0.67$ for $\lambda=1.7$, and is separated from the CE-type insulating phase at $n=0.5$ and the FM insulating phase at $n=0.75^{33}$ by phase separation windows at $T=0.01$. Indeed, $n$ jumps from 0.52 to 0.63 and from 0.67 to 0.75 in the $n$ vs. $\mu$ curve. However, for high temperatures, $n$ monotonically increases with $\mu$ without any discontinuities [see Fig. 2(a) for $T=0.07]$. Figure 2(b) summarizes the $n-T$ phase diagram in the electron density range $n=0.5-0.75$, showing different phases.

We focus on $n=0.65$ that is relevant to the CMR manganites. For this purpose, $\mu$ is varied to fix $n$ for different temperatures [inset of Fig. 2(c)]. The temperature dependence of the magnetic structure factor $S(\mathbf{q})=$ $\frac{1}{N^{2}} \sum_{i j} \mathbf{S}_{\mathbf{i}} \cdot \mathbf{S}_{\mathbf{j}} \mathrm{e}^{i \mathbf{q} \cdot\left(\mathbf{r}_{\mathbf{i}}-\mathbf{r}_{\mathbf{j}}\right)}$ at wave vector $\mathbf{q}=(0,0)(\mathrm{FM}$ correlations) is shown in Fig. 2(c) for different $\Delta$ values. The FM $T_{\mathrm{c}}$ remains the same for $\Delta=0.05$ as compared to $\Delta=0$ and decreases for $\Delta=0.1$. The corresponding resistivity in units of $\hbar a / \pi e^{2}$ ( $a$ : lattice constant) with temperature obtained by calculating the $d c$ limit of the 

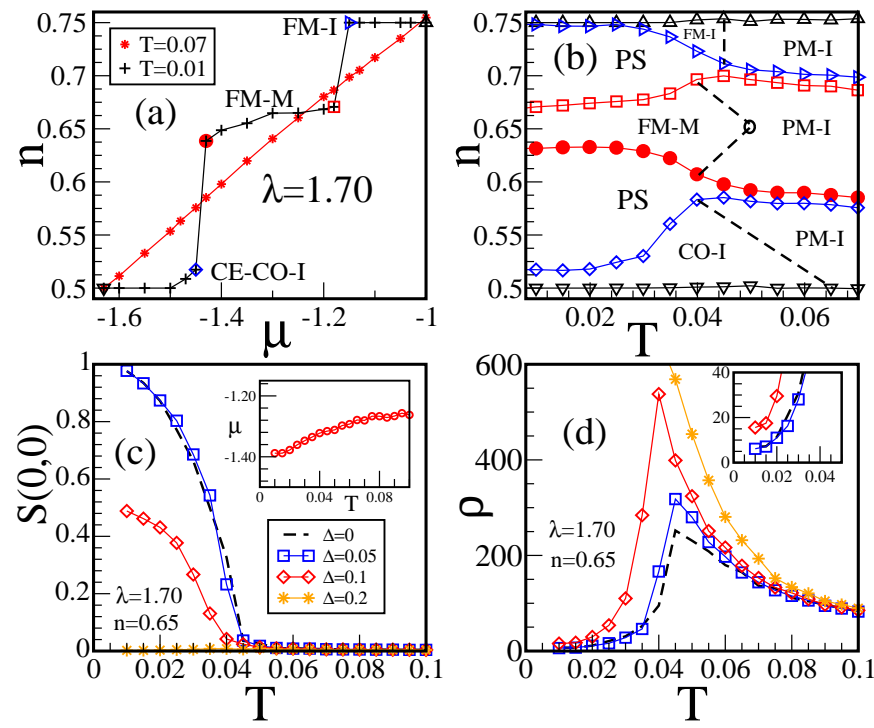

FIG. 2. (Color online) (a) $n$ dependence on $\mu$ for two different temperatures $T=0.01$ and 0.07 . (b) The $n-T$ phase diagram, containing the $\mathrm{CO}$ and FM phases. PS, I, and M denote phase separation, insulator, and metal, respectively. The corresponding phase boundaries at $T=0.01$ are indicated in (a) using the same symbols as in (b). $\Delta=0$ in (a) and (b). $T$ dependence of (c) the FM structure factor $S(0,0)$ and (d) the resistivity $\rho$ in units of $\hbar a / \pi e^{2}$ at $n=0.65$ for different $\Delta$ values indicated in (c). The inset in (c) shows the $\mu$ vs. $T$, required to set the desired $n=0.65$. The inset in (d) shows the enlarged plot at low temperatures. $\lambda=1.7$ for all figures.

conductivity using the Kubo-Greenwood formalism ${ }^{38,39}$ is shown in Fig. 2(d). The magnitude of the resistivity hump around $T_{\mathrm{c}}$ increases with $\Delta$ and its position shifts to the lower temperature. However, the system remains insulating for $\Delta=0.2$ at all temperatures without any long range FM order. The intimate correlation between the onset of the ferromagnetism and the resistivity hump indicates that the metallic and the insulating phases compete each other near $T_{\mathrm{c}}$.

\section{PHASE COMPETITION ABOVE $T_{\mathrm{c}}$}

In order to examine the phase competition, a measure of the volume fraction of the $\mathrm{CO}$ (or FM) regions is necessary because the structure factors in the momentum space are inefficient to probe the local ordering in the real space. Here we calculate the volume fraction of the $n=1 / 2$-type $(\pi, \pi)$ CO nanoclusters $\mathrm{V}(\mathrm{CO})$ from the real space charge distribution. $\mathrm{V}(\mathrm{CO})$ is calculated by counting the fraction of sites for which the local density $n_{i}$ at site $i$, satisfying $n_{i}-0.5 \geq 0.1$, is surrounded by the four nearest neighbor sites $j$ with $0.5-n_{j} \geq 0.1$ or vice versa. Similarly, the volume fraction of the FM nanoclusters, $\mathrm{V}(\mathrm{SO})$, is obtained by calculating the fraction of sites, say $i$, for which all $\mathbf{S}_{\mathrm{i}} \cdot \mathbf{S}_{\mathrm{j}} \geq 0.5$ with the four
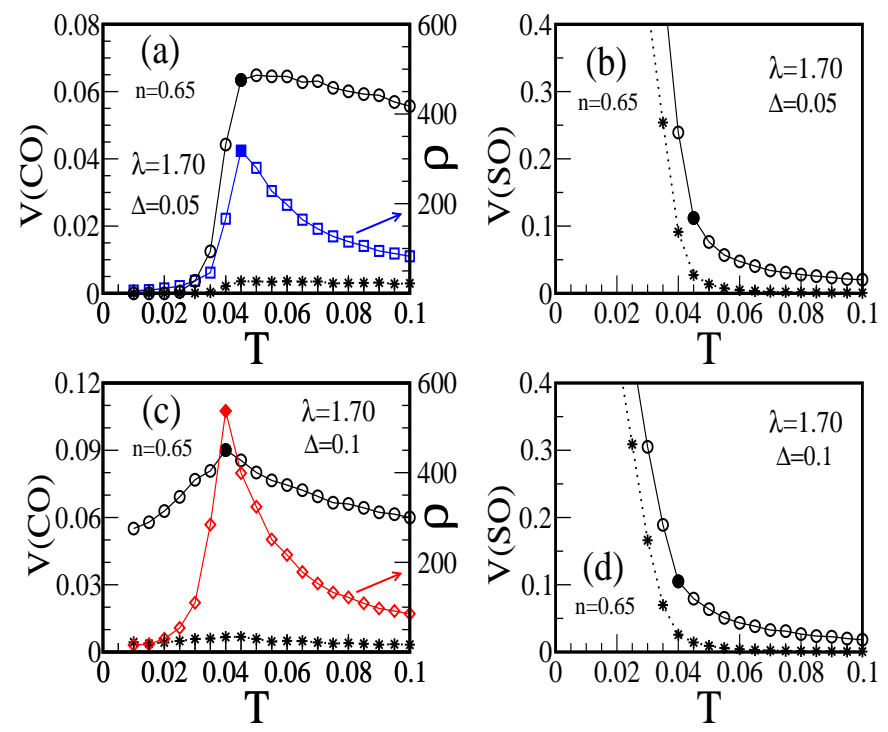

FIG. 3. (Color online) $T$ dependence of the volume fraction of the $\mathrm{CO}$ nanoclusters $\mathrm{V}(\mathrm{CO})$ and resistivity $\rho$ (in units of $\hbar a / \pi e^{2}$ ) for (a) $\Delta=0.05$ and (c) $\Delta=0.1$. The volume fraction of FM nanoclusters $\mathrm{V}(\mathrm{SO})$ vs. $T$ for (b) $\Delta=0.05$ and (d) $\Delta=0.1$. Filled symbols indicate $T_{\mathrm{c}} . n=0.65$ and $\lambda=1.7$ for all figures. See text for dotted lines with star symbols.

nearest neighboring sites $j$.

As shown in Figs. 3(a) and 3(c), $\mathrm{V}(\mathrm{CO})$ for $\Delta=0.05$ and 0.1 , respectively, increases with decreasing $T$ until $T_{\mathrm{c}}$ and decreases thereafter. The volume fraction of the $\mathrm{CO}$ nanoclusters including the next nearest neighboring sites, plotted by dotted lines with star symbols in Figs. 3(a) and $3(\mathrm{c})$, remains very small. Note that $\mathrm{V}(\mathrm{CO})$ decreases to zero for $\Delta=0.05$ but remains finite for $\Delta=0.1$ at low temperatures. The reminiscent of $\mathrm{V}(\mathrm{CO})$ for $\Delta=0.1$ correlates with the fact that the system is not fully FM even at $T=0.01$ where $S(0,0) \sim 0.5$ [see Fig. 2(c)]. This shows the fact that the CO nanoclusters coexist with the $\mathrm{FM}$ regions for $\Delta=0.1$ at low temperatures and as a result the resistivity is larger than in the clean systems, as shown in Fig. 2 (d).

It is apparently clear from Figs. 3(a) and 3(c) that the resistivity increases with $\mathrm{V}(\mathrm{CO})$ until $T_{\mathrm{c}}$ and decreases below it. However, the enhancement of resistivity is steeper than $\mathrm{V}(\mathrm{CO})$, which indicates that electrons moving across the system are not only scattered from the $\mathrm{CO}$ nanoclusters but also from the FM nanoclusters that are possibly present in the system. Therefore, the logical next step is to reveal the presence of FM nanoclusters above $T_{\mathrm{c}}$. For this purpose, we plot $\mathrm{V}(\mathrm{SO})$ in Figs. 3(b) and 3(d). With decreasing $T, \mathrm{~V}(\mathrm{SO})$ increases up to $\sim 0.1$ at $T_{\mathrm{c}}$ and then starts to grow in size. Eventually, the FM nanoclusters merge with each other at low temperatures. This is concluded from the fact that the volume fraction of the FM nanoclusters including the next nearest neighbor sites [dotted line with star symbols in Fig. 3(b) and $3(\mathrm{~d})$ ] remains very small above $T_{\mathrm{c}}$, but 

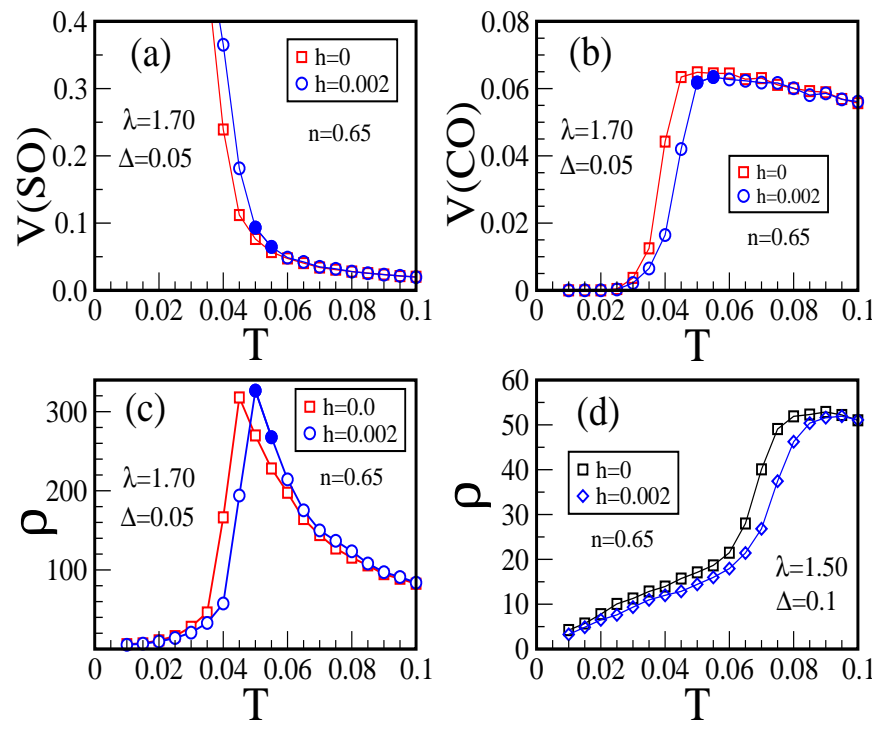

FIG. 4. (Color online) $T$ dependence of the volume fraction of (a) FM nanoclusters $\mathrm{V}(\mathrm{SO})$ and (b) $\mathrm{CO}$ nanoclusters $\mathrm{V}(\mathrm{CO})$ with and without an external magnetic field $h$ for $\lambda=1.7$ and $\Delta=0.05$. Resistivity $\rho$ in units of $\hbar a / \pi e^{2}$ vs. $T$ with and without $h$ for (c) $\lambda=1.7$ and $\Delta=0.05$, and (d) $\lambda=1.5$ and $\Delta=0.1$. Filled symbols in (a) $-(\mathrm{c})$ highlight that $\mathrm{V}(\mathrm{SO})$ and $\mathrm{V}(\mathrm{CO})$ remain unaffected, but the resistivity increases for $T$ $=0.05$ and 0.055 with $h$.

increases below it for both $\Delta=0.05$ and 0.1 . Typically, the strength of disorder is quantified by the variance of the ionic radii of $\mathrm{R}$ and $\mathrm{A}$ ions ${ }^{24,25}$. For example, cation mismatch is very small for $\mathrm{La}_{1-x} \mathrm{Ca}_{x} \mathrm{MnO}_{3}$ (LCMO). In addition, the ground state of LCMO (at $x \sim 1 / 3$ ) is homogeneous $^{2}$. Therefore, we expect that $\Delta=0.05$ is more appropriate for LCMO (see TABLE I), and our calculations show FM nanoclusters coexisting with $\mathrm{CO}$ nanoclusters above $T_{\mathrm{c}}$ and changes to uniform FM metal at low temperatures.

We now apply a very small external magnetic field $h$ to further confirm the phase coexistence above $T_{\mathrm{c}}$. For $h=$ 0.002 , the $T_{\mathrm{c}}$ increases from 0.045 to 0.05 [Figs. 4(a) and (b)]. For $T \geq 0.05, \mathrm{~V}(\mathrm{CO})$ and $\mathrm{V}(\mathrm{SO})$ remain unaffected with $h$. This indicates that the applied magnetic field does not affect the $\mathrm{CO}$ regions, but only aligns spins in different FM nanoclusters without increasing their size, as shown schematically in Fig. 1(c). For $h=0$, magnetic nanoclusters are randomly oriented. Therefore, in addition to the CO nanoclusters, the up-spin (down-spin) electrons scatters from the down-spin (up-spin) oriented magnetic nanoclusters and the current is carried equally in both spin channels. A small external magnetic field aligns the magnetic nanoclusters, say in the up direction, which decreases the current from the down-spin electrons. This results in an overall enhancement of the resistivity, as shown in Fig. 4(c) using filled symbols, and substantiates the presence of the FM nanoclusters above $T_{\mathrm{c}}$, in addition to the CO nanoclusters. Such enhancement of resistivity in a magnetic field due to the
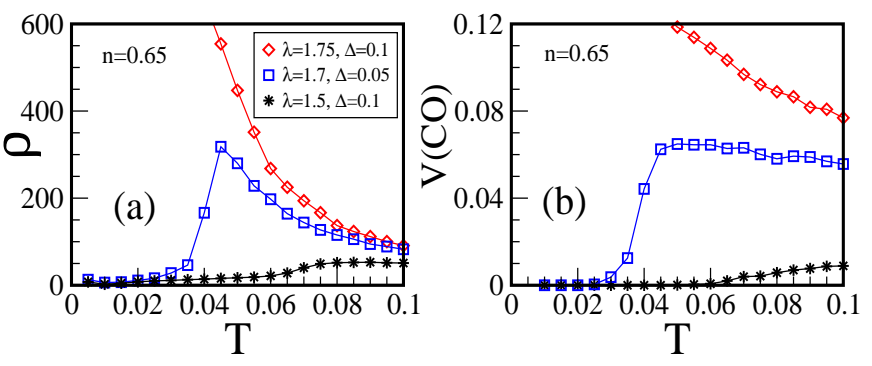

FIG. 5. $T$ dependence of (a) the resistivity $\rho$ in units of $\hbar a / \pi e^{2}$ and (b) $\mathrm{V}(\mathrm{CO})$ for $\lambda=1.75,1.7$, and 1.5. $\Delta$ values are mentioned in figure. Legends in (a) and (b) are the same.

presence of FM and $\mathrm{CO}$ nanoclusters also resemble with FM-metallic/insulating multilayers in which spins in the individual FM layers are randomly oriented ${ }^{40-42}$. In addition, we find in Fig. 4(d) that the resistivity for $\lambda=1.5$ and $\Delta=0.1$ does not increases in a small external magnetic field $(h=0.002)$ around $T_{\mathrm{c}}(=0.075)$ because of the absence of CO nanoclusters (discussed below).

Our overall results show that the phase competition between $\mathrm{CO}$ and FM nanoclusters above $T_{\mathrm{c}}$ induces the resistivity hump, which is observed in LCMO. However, for large bandwidth manganites, e.g. LSMO (for an abbreviation, see Table I), the resistivity hump diminishes around $T_{\mathrm{c}}$ [see Fig. 1(b)]. In order to understand this, we show the resistivity and $\mathrm{V}(\mathrm{CO})$ for $\lambda=1.5,1.7$, and 1.75 in Fig. 5(a) and Fig. 5(b), respectively. Recall that $\lambda$ is measured in units of $t$, and thus smaller $\lambda$ corresponds to larger bandwidth or vice versa. For $\lambda=1.5$ (1.75), the ground state is FM metallic (CE-type insulating) both at $n=0.65$ and $n=0.5$, similar to LSMO (PCMO) [see Fig. 1(a)]. A qualitative estimation of $\lambda$ for different manganites is listed in Table I. For $\lambda=1.5, \mathrm{~V}(\mathrm{CO})$ is very small and monotonically decreases with decreasing $T$, resulting in no resistivity hump. This also suggests that the CO nanoclusters above $T_{\mathrm{c}}$ at $n=0.65$ appears only for specific systems for which the ground state at $n \sim 0.5$ is a CE-type phase. LSMO does not have the CE-type phase at any hole doping unlike LCMO [see Fig. 1(a)] and as a result the resistivity hump vanishes ${ }^{43}$. For $\lambda=1.75$ i.e. small bandwidth manganites such as PCMO V(CO) increases with decreasing $T$ and eventually an insulating state appears at low temperatures. We have considered $\Delta=0.1$ for $\operatorname{LSMO}(\lambda=1.5)$ and $\operatorname{PCMO}(\lambda=1.75)$ as the disorder strength is larger than $\operatorname{LCMO}(\lambda=1.7)$.

\section{COMPARISON WITH EXPERIMENTS}

We turn now to explore the impact of disorder. As shown in Fig. 6(a), the resistivity hump appears and the magnitude increases with $\Delta$, even for $\lambda=1.5$, and its position shifts to lower temperature similar to the case for $\lambda=1.7$ [Fig. 2(d)]. However, relatively large $\Delta$ is required to convert the FM metal to a disorderassisted insulator for smaller $\lambda$. Also, for $\Delta=0.1$, 

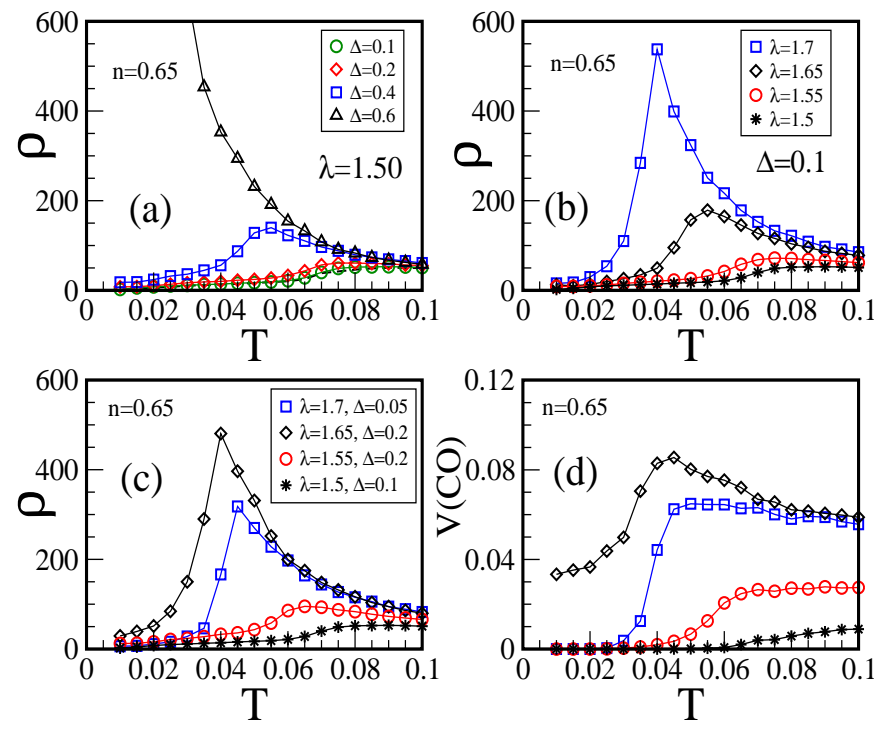

FIG. 6. (a)-(c): $T$ dependence of the resistivity $\rho$ in units of $\hbar a / \pi e^{2}$ for different parameters (indicated in the figures). In (a), the dashed line is for $\Delta=0$. (d): Temperature dependence of $\mathrm{V}(\mathrm{CO})$ for the same set of parameters used in (c). Legends in (c) and (d) are the same.

$T_{\mathrm{c}}$ increases but the magnitude of resistivity hump decreases with decreasing $\lambda$ (equivalent to increasing the bandwidth) [see Fig. 6(b)]. This is similar to the experiments shown schematically in Fig. 1(b) ${ }^{43-45}$ except for NSMO. Although LCMO has the smaller bandwidth than NSMO, the magnitude of the resistivity hump for LCMO is smaller than NSMO. In order to explain the observed resistivity trend properly, one needs to estimate $\Delta$ values correctly, at least qualitatively. Due to large mismatch between $\mathrm{R}$ and $\mathrm{A}$ radii, the disorder strength in NSMO is larger than LCMO and thus relatively larger $\Delta$ must be set (see Table I). As discussed earlier, $\Delta$ for LCMO is minimal, while it increases for LSMO and increases further for NSMO and PSMO. Figure 6(c) shows the resistivity vs. temperature for four combinations of $\lambda$ and $\Delta$ values, corresponding to four different manganites (NSMO, LCMO, PSMO, and LSMO) listed in Table I. Resistivity curves in Fig. 6(c) qualitatively agree with the experiments ${ }^{43-45}$ shown schematically in Fig. 1(b). This shows that the disorder also plays an important role to understand the experimental results systematically. V(CO) plotted in Fig. 6(d) shows a similar trend and clarifies the one-to-one correspondence between the resistivity and the volume fraction the $\mathrm{CO}$ nanoclusters in CMR manganites.

\section{CONCLUSION}

In summary, based on the two-band model, we have provided a systematic study to demonstrate the phase competitions between the $\mathrm{CO}$ and the FM nanoclusters above $T_{\mathrm{c}}$ in CMR manganites. The resistivity increases due to the enhancement of the volume fraction of the $n=1 / 2$-type $(\pi, \pi) \mathrm{CO}$ and FM nanoclusters, with decreasing $T$ until $T_{\mathrm{c}}$. The FM nanoclusters start to grow and merge, and wins the competition below $T_{\mathrm{c}}$, leading to the sharp drop in the resistivity. The CO nanoclusters do not form in large bandwidth manganites and as a result the resistivity hump vanishes. Our calculations establish a simple yet complete pathway to understand the phase competitions in CMR manganites.

\section{ACKNOWLEDGEMENTS}

We acknowledge use of TCMP computer cluster at SINP and our discussions with P. Majumdar and S. K. Das. A part of the numerical calculations have been done using the computational resource on HOKUSAI GreatWave supercomputer allocated by RIKEN Advanced Center for Computing and Communication (ACCC).
* email: kalpataru.pradhan@saha.ac.in

† email: yunoki@riken.jp

1 Colossal Magnetoresistive Oxides, edited by Y. Tokura (Gordon and Breach, New York, 2000).

2 Nanoscale Phase Separation and Colossal Magnetoresistance: The Physics of Manganites and Related Compounds, E. Dagotto (Springer, 2003).

3 E. Dagotto, T. Hotta, and A. Moreo, Phys. Rep. 344, 1 (2001).

${ }^{4}$ Y. Tokura, Rep. Prog. Phys. 69, 797 (2006).

5 M. Uehara, S. Mori, C. H. Chen, and S.-W. Cheong, Nature (London) 399, 560 (1999).

6 M. Faeth, S. Freisem, A. A. Menovsky, Y. Tomioka, J. Aarts, and J. A. Mydosh, Science 285, 1540 (1999).

7 J. M. Zuo and J. Tao, Phys. Rev. B 63, 060407R (2001).
8 T. Y. Koo, V. Kiryukhin, P. A. Sharma, J. P. Hill, and S. W. Cheong, Phys. Rev. B 64, 220405R (2001).

9 L. Zhang, C. Israel, A.Biswas, R. L. Greene, and A. de. Lozanne, Science 298, 805 (2002).

10 D. D. Sarma, Dinesh Topwal, U. Manju, S. R. Krishnakumar, M. Bertolo, S. La Rosa, G. Cautero, T. Y. Koo, P. A. Sharma, S.-W. Cheong, and A. Fujimori, Phys. Rev. Lett. 93, 097202 (2004).

11 S. Chaudhuri, R. C. Budhani, Jiaqing He, and Yimei Zhu, Phys. Rev. B 76, 132402 (2007).

12 J. Tao, D. Niebieskikwiat, M. Varela, W. Luo, M. A. Schofield, Y. Zhu, M. B. Salamon, J. M. Zuo, S. T. Pantelides, and S. J. Pennycook, Phys. Rev. Lett. 103, 097202 (2009).

13 Y. Murakami, H. Kasai, J. J. Kim, S. Mamishin, D. Shindo, S. Mori, and A. Tonomura, 
Nature Nanotechnology 5, 37 (2010).

14 J. Tao, D. Niebieskikwiat, Q. Jie, M. A. Schofield, L. Wu, Q. Li, and Y. Zhu, Proc. Natl. Acad. Sci. USA 108, 20941 (2011).

15 Mark H. Burkhardt, M. A. Hossain, S. Sarkar, Y.-D. Chuang, A. G. Cruz Gonzalez, A. Doran, A. Scholl, A. T. Young, N. Tahir, Y. J. Choi, S.-W. Cheong, H. A. Duerr, and J. Stoehr Phys. Rev. Lett. 108, 237202 (2012).

16 Y. Horibe, S. Mori, T. Asaka, Y. Matsui, P. A. Sharma, T. Y. Koo, S. Guha, C. H. Chen and S.-W. Cheong, Europhys. Lett. 100, 67007 (2013).

17 N. S. Bingham, P. Lampen, M. H. Phan, T. D. Hoang, H. D. Chinh, C. L. Zhang, S. W. Cheong, and H. Srikanth Phys. Rev. B 86, 064420 (2013).

18 R. Rawat, Pallavi Kushwaha, Dileep K. Mishra, and V. G. Sathe, Phys. Rev. B 87, 064412 (2013).

19 L. Z. Liang, L. Li, H. Wu, and X. H. Zhu, Nanoscale Res. Lett. 9, 325 (2014).

20 J. Shao, H. Liu, K. Zhang, Y. Yu, W. Yu, H. Lin, J. Niu, K. Du, Y. Kou, W. Wei, F. Lan, Y. Zhu, W. Wang, J. Xiao, L. Yin, E. W. Plummer, and J. Shen, Proc. Natl. Acad. Sci. USA 1139228 (2016).

${ }^{21}$ K. Zhang, L. Li, H. Li, Q. Feng, N. Zhang, L. Cheng, X. Fan, Y. Hou, Q. Lu, Z. Zhang, and C. Zeng, Nano Letters 17, 1461 (2017).

22 C. Martin, A. Maignan, M. Hervieu, and B. Raveau, Phys. Rev. B 60, 12191 (1999).

${ }^{23}$ R. Kajimoto, H. Yoshizawa, Y. Tomioka, and Y. Tokura, Phys. Rev. B 66, 180402 (2002).

24 H. Y. Hwang, S-W. Cheong, P. G. Radaelli, M. Marezio, and B. Batlogg, Phys. Rev. Lett. 75, 914 (1995).

25 L. M. Rodriguez-Martinez and J. P. Attfield, Phys. Rev. B 63, 024424 (2000).

26 A. Moreo, S. Yunoki, and E. Dagotto, Science 283, 2034 (1999).

27 E. Dagotto, New. J. Phys. 7, 67 (2005).
28 C. Sen, G. Alvarez, and E. Dagotto, Phys. Rev. Lett. 105, 097203 (2010).

29 C. Sen, S. Liang, and E. Dagotto, Phys. Rev. B 85, 174418 (2012).

30 S. Yunoki, T. Hotta, and E. Dagotto, Phys. Rev. Lett. 84, 3714 (2000).

31 S. Kumar, A. P. Kampf, and P. Majumdar, Phys. Rev. Lett. 97, 176403 (2006).

32 K. Pradhan, A. Mukherjee, and P. Majumdar, Phys. Rev. Lett. 99, 147206 (2007).

33 K. Pradhan, A. Mukherjee, and P. Majumdar, Europhys. Lett. 84, 37007 (2008).

34 S. Yunoki, J. Hu, A. L. Malvezzi, A. Moreo, N. Furusaki, and E. Dagotto, Phys. Rev. Lett. 80, 845 (1998).

35 E. Dagotto, S. Yunoki, A. L. Malvezzi, A. Moreo, J. Hu, S. Capponi, D. Poilblanc, and N. Furukawa, Phys. Rev. B 58, 6414 (1998).

36 S. Yunoki, A. Moreo, and E. Dagotto, Phys. Rev. Lett. 81, 5612 (1998).

$37 \mathrm{~S}$ Kumar and P. Majumdar, Eur. Phys. J. B 50, 571 (2006).

38 G. D. Mahan, Quantum Many Particle Physics (Plenum Press, New York, 1990).

39 S. Kumar and P. Majumdar, Europhys. Lett. 65, 75 (2004).

40 Z. G. Sheng, W. H. Song, Y. P. Sun, J. R. Sun, and B. G. Shen, Appl. Phys. Lett. 87, 032501 (2005).

41 T. F. Zhou, G. Li, N. Y. Wang, B. M. Wang, X. G. Li, and Y. Chen, Appl. Phys. Lett. 88, 232508 (2006).

${ }^{42}$ K. Pradhan and A. P. Kampf, Phys. Rev. B 87, 115152 (2013).

43 J. M. D. Coey, M. Viret, L. Ranno, and K. Ounadjela, Phys. Rev. Lett. 75, 3910 (1995).

44 E. Saitoh, Y. Okimoto, Y. Tomioka, T. Katsufuji, and Y. Tokura, Phys. Rev. B 60, 10362 (1999).

45 N. Furukawa and Y. Motome, J. Phys. Soc. Jpn. 74, 203 (2005). 